\title{
Membranous and cytoplasmic expression of epidermal growth factor receptor in metastatic pancreatic ductal adenocarcinoma
}

\author{
TAKAHIRO EINAMA ${ }^{1,6}$, SHIGETO UEDA ${ }^{2}$, HITOSHI TSUDA ${ }^{3,7}$, KAZUHIRO OGASAWARA $^{5}$, \\ KAZUO HATSUSE ${ }^{4}$, OSAMU MATSUBARA ${ }^{3}$, SATORU TODO ${ }^{1}$ and JUNJI YAMAMOTO ${ }^{4}$
}

\author{
${ }^{1}$ Department of General Surgery, Hokkaido University, Graduate School of Medicine, Sapporo; ${ }^{2}$ Medical Service School, \\ National Defense Force, Tokyo; Departments of ${ }^{3}$ Basic Pathology and ${ }^{4}$ Surgery, National Defense Medical College, \\ Saitama; ${ }^{5}$ Department of Surgery, Japan Labor Health and Welfare Organization Kushiro Rosai Hospital, Kushiro, \\ Hokkaido; ${ }^{6}$ Division of Gastroenterological and General Surgery, Department of Surgery, \\ Asahikawa Medical University, Hokkaido, Japan
}

Received January 13, 2012; Accepted February 27, 2012

DOI: 10.3892/etm.2012.518

\begin{abstract}
Recent studies indicate the clinical significance of the cellular localization of epidermal growth factor receptor (EGFR) in a variety of cancer types. Internalization of activated EGFR is reported to be closely associated with patient prognosis. This study investigated the clinical significance of the immunohistochemical localization of EGFR in patients with metastatic pancreatic cancers compared to those with surgically resected pancreatic cancers. Using 44 surgically resected primary pancreatic cancers and 40 primary or metastatic tumors from 20 autopsied patients with far advanced pancreatic cancers, the incidence of membranous and cytoplasmic EGFR overexpression was compared between primary tumors and far advanced tumors by immunohistochemistry using the Dako EGFR pharmDx ${ }^{\mathrm{TM}}$ kit, a global standard kit for EGFR assay. In the 44 surgically resected cancers, 13 (30\%) exhibited membranous overexpression of EGFR, comprising 1 case $(2 \%)$ with score $3+$ and 12 cases $(27 \%)$ with score $2+$ and $10(23 \%)$ exhibited cytoplasmic overexpression of EGFR. In the 40 tumors at a far advanced stage, the percentage of samples exhibiting positivity for membranous and cytoplasmic EGFR overexpression was 48\% (19 of 40) comprising 7 (18\%) with score $2+$ and 12 (30\%) with score $3+$ and $33 \%$ (13 of 40), respectively. The far advanced tumors tended to show membranous and cytoplasmic EGFR overexpression more frequently than the surgically resected tumors, although the difference was not significant. These findings suggest that membranous and cytoplasmic overexpression of EGFR may be indicative of the potential aggressiveness of pancreatic cancers.
\end{abstract}

Correspondence to: Dr Hitoshi Tsuda, ${ }^{7}$ Present address: Pathology Section, Clinical Laboratory Division, National Cancer Center Hospital, 5-1-1 Tsukiji, Chuo-ku, Tokyo 104-0045, Japan E-mail: hstsuda@ncc.go.jp

Key words: epidermal growth factor receptor, metastatic pancreatic cancer, cytoplasmic expression

\section{Introduction}

Despite recent advances in diagnostic and therapeutic techniques, pancreatic carcinoma is one of the most lethal malignancies among cancers. The 5-year survival rate of patients having primary pancreatic cancer after complete resection does not reach $15 \%$ (1), while the overall 5-year survival rates in patients having inoperable pancreatic cancer are desperately low, ranging from 0.4 to $4 \%(2,3)$.

Currently, gemcitabine is a key drug not only for treating advanced pancreatic cancer (4) but also as an adjuvant chemotherapy regimen for resectable pancreatic cancer $(5,6)$. Furthermore, molecular targeting of epidermal growth factor receptor (EGFR) or vascular endothelial growth factor (VEGF) has recently been developed to treat these lesions $(7,8)$. Moore et al reported in a phase III trial of patients with advanced pancreatic cancer, that erlotinib, a tyrosine kinase inhibitor of EGFR, in combination with gemcitabine was superior to gemcitabine alone when progression-free and overall survival were compared between the two groups (8).

EGFR, one of the tyrosine kinase receptors of the ErbB family, is reported to be expressed immunohistochemically in $10-30 \%$ of patients with solid tumors including pancreatic carcinoma $(9,10)$. Tyrosine phosphorylation in EGFR protein in cancer cells leads to activation of several downstream intracellular substrates and plays a pivotal role in tumor proliferation, invasion and metastasis (11). Recent studies have suggested that the EGFR gene copy number and expression obtained by fluorescence in situ hybridization (FISH) and immunohistochemistry (IHC) predict the clinical response of a tumor to gefitinib, a tyrosine kinase inhibitor of EGFR, in patients with non-small cell lung cancer (12-14). Furthermore, recent studies have found that mutations of the EGFR gene at the restricted region, e.g., exon 19 and exon 21, were closely correlated with response to gefitinib therapy (15-20). However, the relevance of EGFR expression in pancreatic cancer with therapeutic response has remained to be verified (8).

Although immunohistochemical expression of EGFR can also be recognized as positive membranous staining, 
Table I. Clinicopathological characteristics of the patients and tumors.

\begin{tabular}{|c|c|c|c|c|}
\hline \multirow[b]{2}{*}{ Parameter } & \multirow{2}{*}{$\frac{\text { Surgically resected cancers }(\mathrm{n}=44)}{\mathrm{n}(\%)}$} & \multicolumn{3}{|c|}{ Far advanced cancers $(n=20)$} \\
\hline & & & $\mathrm{n}(\%)$ & \\
\hline Age (mean \pm SD, years) & $63.3 \pm 3.7$ & & $57.3 \pm 5.7$ & \\
\hline$<65$ & $23(52)$ & & $13(65)$ & \\
\hline$\geq 65$ & $21(47)$ & & $7(35)$ & \\
\hline \multicolumn{5}{|l|}{ Gender } \\
\hline Male & $34(77)$ & & $16(80)$ & \\
\hline Female & $10(23)$ & & $4(20)$ & \\
\hline \multicolumn{5}{|l|}{ Tumor site } \\
\hline Head & $36(82)$ & & $12(60)$ & \\
\hline Body and/or tail & $8(18)$ & & $8(40)$ & \\
\hline \multicolumn{5}{|l|}{ Stage } \\
\hline I & 1 (2) & & & \\
\hline II & $32(73)$ & & & \\
\hline III & $8(18)$ & & & \\
\hline IV & $3(7)$ & & & \\
\hline \multicolumn{5}{|l|}{ Grade } \\
\hline 1 & $12(27)$ & $5(25)^{\mathrm{a}}$ & & $2(10)^{b}$ \\
\hline 2 & $28(64)$ & $3(15)^{\mathrm{a}}$ & & $9(45)^{b}$ \\
\hline 3 & 4 (9) & $12(60)^{\mathrm{a}}$ & & $9(45)^{b}$ \\
\hline Median survival (mean $\pm \mathrm{SD}$, month) & $24.5 \pm 10.3$ & & & \\
\hline
\end{tabular}

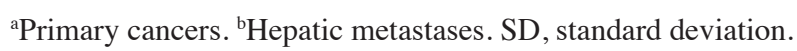

cytoplasmic expression of EGFR can frequently be observed in cancer cells of the pancreas. We previously reported that high cytoplasmic expression of EGFR in primary pancreatic cancer was significantly correlated with higher histological grade and poorer survival (10), suggesting that cytoplasmic EGFR expression could indicate a potentially aggressive or metastatic feature of pancreatic cancer. However, it is unclear whether localization of EGFR expression differs between primary and metastatic sites of pancreatic cancers at surgically resectable stages and those at inoperable far advanced stages.

The present study compared immunohistochemically the levels and localization of EGFR expression between surgically resected primary pancreatic cancers and far advanced cancers obtained at autopsy, in order to clarify the clinical impact of membranous and cytoplasmic EGFR overexpressions in far advanced pancreatic cancers.

\section{Materials and methods}

Patients and tumor specimens. This study was performed with approval by the Internal Review Board on Ethical Issues of the National Defense Medical College, Japan. The subjects of this study were 44 patients who underwent surgery with curative intent for primary pancreatic cancers between 1987 and 2000 at the National Defense Medical College Hospital, Tokorozawa, Japan. The clinicopathological characteristics of these cases are summarized in Table I.
The mean patient age was 63.3 years $[ \pm 3.7$ standard deviation (SD)]. Thirty-four (77.3\%) were men and 10 (22.7\%) were women. More than $80 \%$ of tumors were located in the head of the pancreas. As for stage, approximately $90 \%$ of the patients were assigned to stage II or stage III (21). Histologically, all 44 patients had invasive ductal adenocarcinoma of the pancreas, and the majority of the patients had moderately differentiated tubular adenocarcinoma. The median survival time was 24.5 months $( \pm 10.3 \mathrm{SD})$.

In addition, a total of 40 tumor specimens from primary sites and hepatic metastatic sites were obtained at autopsy from 20 patients who had died of inoperable far advanced pancreatic cancer between 1980 and 2001 at the same hospital (Table I).

Using these tumor specimens from a total of 64 patients, formalin-fixed paraffin-embedded tissue blocks were prepared, and sections were cut and stained with hematoxylin and eosin (H\&E) for routine histopathological examination. Because surgically resected specimens had been cut routinely for pathology specimens once a weak periodically, the duration of formalin fixation of the surgically resected specimens varied from 1 to 6 days. Likewise, the duration of formalin fixation of the autopsied tissues varied from 1 to 6 days. All specimens were diagnosed as ductal adenocarcinomas of the pancreas. After a histological review of the sections by three observers (T.E., H.T. and S.U.), a representative tissue block was selected from each surgically resected primary tumor, each primary tumor obtained by autopsy, and each metastatic tumor obtained 

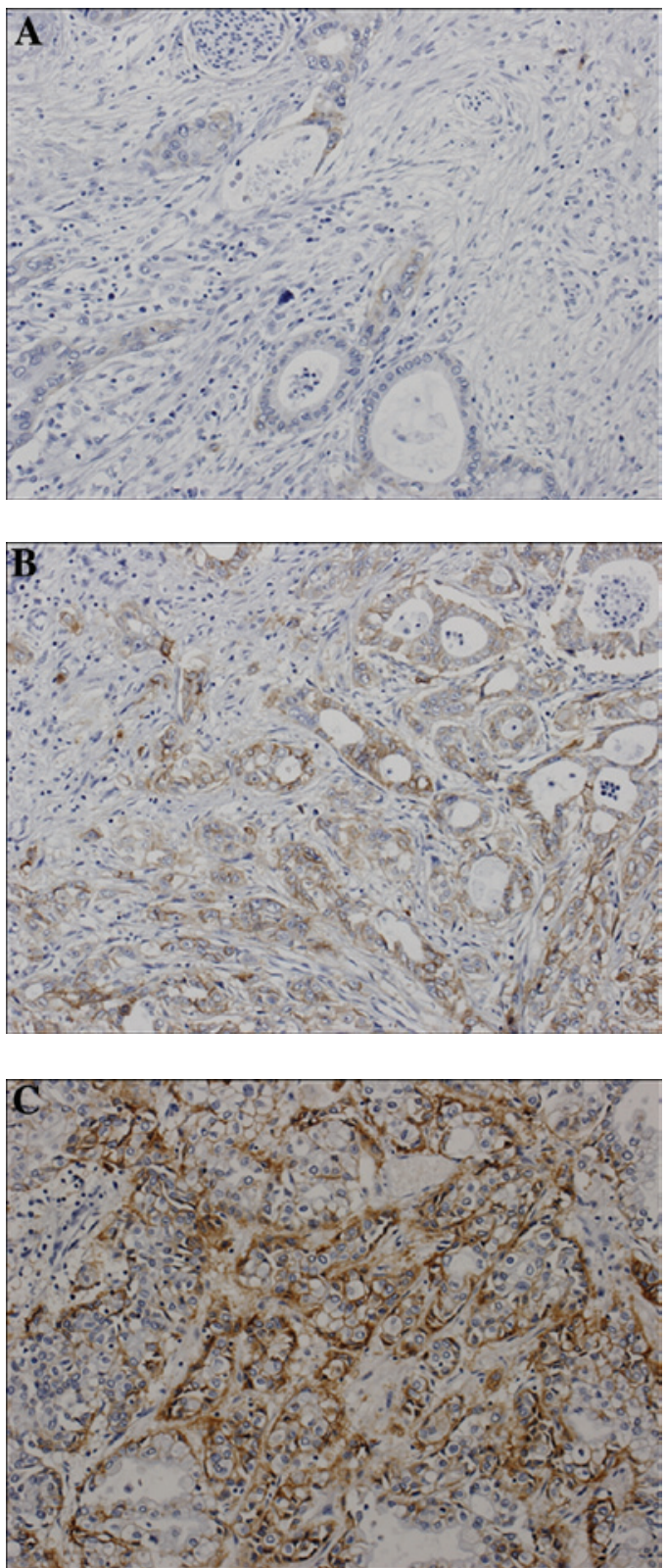

Figure 1. Representative cases of pancreatic ductal adenocarcinoma showing scores of 1+, 2+ and 3+ for membranous EGFR expression. (A) Score 1+, incomplete membrane staining is weakly visible. (B) Score $2+$, the entire circumference of the cell membrane is weakly stained. (C) Score $3+$, the entire circumference of the cell membrane is heavily stained. Immunoperoxidase stain, x200.

by autopsy. These tumor tissue blocks were subjected to immunohistochemical studies.

Histological classification. The three observers graded the degree of tumor differentiation. Tumor differentiation was classified into Grade 1 (well-differentiated type), Grade 2 (moderately differentiated type) and Grade 3 (poorly differentiated type), according to the degree of tubular formation (21). The grade of each primary cancer was defined according to the findings in the widest area of the representative section of the cancer.

Immunohistochemistry. Immunohistochemical staining for EGFR was performed using the EGFR pharmDx ${ }^{\mathrm{TM}}$ kit (Dako,
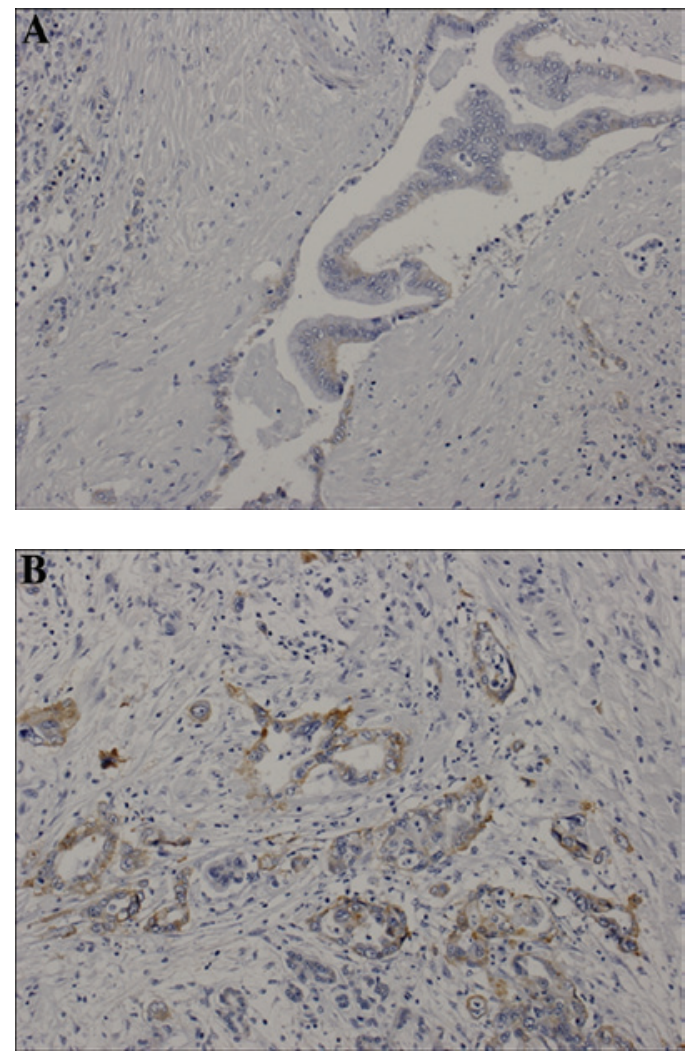

Figure 2. Representative cases of pancreatic ductal adenocarcinoma showing scores of $1+$ and $2+$ for EGFR cytoplasmic expression. (A) Score 1+, faint diffuse cytoplasmic staining is detected. (B) Score $2+$, moderate to strong cytoplasmic staining and strong granular staining is observed. Immunoperoxidase stain, $\mathrm{x} 200$.

Carpinteria, CA, USA), a global standard kit for EGFR assay approved by the US Food and Drug Administration (US FDA). Sections were deparaffinized in two sequential xylene baths (5 $\mathrm{min}), 100 \%$ ethanol ( $3 \mathrm{~min}$ ) and $95 \%$ ethanol ( $3 \mathrm{~min}$ ), followed by a 5 -min single wash in wash-buffer solution (Dako). Subsequently, at room temperature, the section was rinsed in wash-buffer for $5 \mathrm{~min}$, incubated in proteinase $\mathrm{K}$ solution (Dako) for $5 \mathrm{~min}$, rinsed again in the wash-buffer for $5 \mathrm{~min}$, incubated in peroxidase blocking agent for $5 \mathrm{~min}$, rinsed, incubated with the primary EGFR antibody or negative control reagent for $30 \mathrm{~min}$, rinsed, incubated with visualization reagent for $30 \mathrm{~min}$, rinsed twice with the buffer, incubated with substrate chromogen solution for $5 \mathrm{~min}$ and finally rinsed again with the buffer. Slides were counterstained with hematoxylin and rinsed gently in reagent quality water. The positive and negative controls used were formalin-fixed, paraffin-embedded pellets of HT-29 and CAMA-1 cell lines, which expressed and did not express EGFR, respectively (Dako).

Immunohistochemical evaluation. Immunohistochemical evaluation was performed for both the cell membrane and cytoplasm, separately, for the primary or metastatic carcinoma samples. The level of membranous EGFR expression was stratified into 4 groups (scores $0,1+, 2+$ and $3+$ ) according to the criteria for the HER2 test (HercepTest) (22). In detail, when membranous staining was observed in $<10 \%$ of the tumor 
Table II. EGFR immunostaining in the surgically resected cancers and the far advanced cancers obtained at autopsy.

\begin{tabular}{|c|c|c|c|c|c|c|c|c|}
\hline & \multicolumn{8}{|c|}{ No. of cases $(\%)$} \\
\hline & \multirow[b]{2}{*}{ Total } & \multicolumn{4}{|c|}{ Membranous EGFR reactivity } & \multicolumn{3}{|c|}{ Cytoplasmic EGFR reactivity } \\
\hline & & 0 & $1+$ & $2+$ & $3+$ & 0 & $1+$ & $2+$ \\
\hline Surgically resected cancers & 44 & $24(55)$ & $7(16)$ & $12(27)$ & $1(2)$ & $22(50)$ & $12(27)$ & $10(23)$ \\
\hline Far advanced cancers & 40 & $6(15)$ & $15(38)$ & $7(18)$ & $12(30)$ & $4(10)$ & $23(58)$ & $13(33)$ \\
\hline Primary cancers ${ }^{\mathrm{a}}$ & 20 & $3(15)$ & $9(45)$ & $3(15)$ & $5(25)$ & $2(10)$ & $13(65)$ & $5(25)$ \\
\hline Hepatic metastases ${ }^{\mathrm{a}}$ & 20 & $3(15)$ & $6(30)$ & $4(20)$ & $7(35)$ & $2(10)$ & $10(50)$ & $8(40)$ \\
\hline
\end{tabular}

${ }^{\text {an }}$ o significant difference between membranous and cytoplasmic EGFR reactivity.

Table III. Expression of EGFR stratified according to histological grading between the surgically resected cancers and the far advanced cancers obtained at autopsy.

\begin{tabular}{|c|c|c|c|c|c|}
\hline \multirow[b]{2}{*}{ Histological grade } & \multirow[b]{2}{*}{ Total } & \multicolumn{4}{|c|}{ No. of tumors (\%) } \\
\hline & & $\begin{array}{c}\text { Membranous } \\
\text { EGFR overexpression }\end{array}$ & P-value ${ }^{a}$ & $\begin{array}{c}\text { Cytoplasmic } \\
\text { EGFR overexpression }\end{array}$ & P-value ${ }^{a}$ \\
\hline Surgically resected cancers & 44 & $13(30)^{\mathrm{b}}$ & & $10(23)^{\mathrm{c}}$ & \\
\hline Grade 1 & 12 & 1 (8) & 0.07 & $1(8)$ & 0.2 \\
\hline Grade $2 / 3$ & 32 & $12(38)$ & & $9(28)$ & \\
\hline Far advanced cancers & 40 & $19(48)^{b}$ & & $13(33)^{\mathrm{c}}$ & \\
\hline Grade 1 & 7 & $3(43)$ & 0.8 & $1(14)$ & 0.3 \\
\hline Grade $2 / 3$ & 33 & $16(48)$ & & $12(36)$ & \\
\hline
\end{tabular}

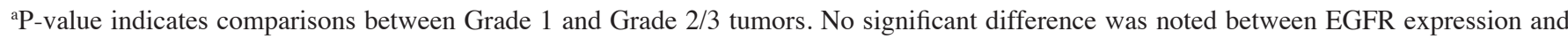
histological grade (Grade 1 vs. $2 / 3$ ). ${ }^{b} \mathrm{P}=0.09$, statistically significant difference between surgically resected and advanced cancers. ${ }^{\mathrm{c}} \mathrm{P}=0.3$, statistically significant difference between surgically resected and advanced cancers.

cells, a score of 0 was assigned, regardless of the intensity of the staining. If faint or barely perceptible membranous staining was detected in $>10 \%$ of the tumor cells, a score of $1+$ was assigned. Scores of $2+$ and $3+$ were assigned when weak to moderate staining and strong staining, respectively, were observed on the entire membrane in $>10 \%$ of the tumor cells (Fig. 1). Cases showing a score of $2+$ or $3+$ were defined as showing overexpression.

Cytoplasmic staining was divided into 3 grades $(0,1+$ and $2+)$, as grading of the intensity of the immunoreaction was difficult for the cytoplasm. The level of cytoplasmic staining was categorized as follows: when cytoplasmic staining was observed in $<10 \%$ of the tumor cells, a score of 0 was assigned. If faint or barely perceptible cytoplasmic staining was detected in $>10 \%$ of tumor cells, a score of $1+$ was assigned. A score of 2+ was assigned when moderate or strong staining, respectively, was observed in $>10 \%$ of the tumor cells. Cytoplasmic granular staining was also scored as $2+$. Cases showing a score of $2+$ were judged as showing overexpression (Fig. 2).

Statistical analysis. We used the $\chi^{2}$ test or Fisher's exact test to determine the correlation between EGFR expression and histological grade. Differences were considered to indicate statistical significance at a P-value $<0.05$. All statistical analyses were performed using Statview 5.0 software (SAS Institute Inc., Cary, NC, USA).

\section{Results}

The expression profiles of membranous and cytoplasmic EGFR in both surgically resected cancers and far advanced cancers obtained at autopsy are shown in Table II. In the 44 surgically resected cancers, 13 (30\%) exhibited membranous overexpression of EGFR, comprising 1 case $(2 \%)$ of score $3+$ and 12 cases $(27 \%)$ of score $2+$ and $10(23 \%)$ exhibited cytoplasmic overexpression of EGFR.

In the primary tumors in the 20 far advanced cancers, the percentage of samples with positivity for membranous EGFR overexpression was $40 \%$, (8 of 20), comprising 3 cases $(15 \%)$ of score $2+$ and 5 cases $(25 \%)$ of score $3+$, and the percentage of samples showing positivity for cytoplasmic EGFR overexpresion was $25 \%$ (5 of 20). In the hepatic metastases in the 20 far advanced cancers, the positivity of membrane EGFR overexpression was $55 \%$, (11 of 20), comprising 4 cases $(20 \%)$ of score $2+$ and 7 cases $(35 \%)$ of score $3+$, and the positivity of cytoplasmic EGFR overexpresion was $40 \%$ (8 of 20 ). 
In a total of 40 tumors at a far advanced stage, the percentage of samples showing positivity for membranous EGFR overexpression was $48 \%$ (19 of 40) comprising 7 cases (18\%) of score $2+$ and 12 cases $(30 \%)$ of score $3+$, and the percentage of samples showing positivity for cytoplasmic EGFR overexpresion was $33 \%$ (13 of 40). Therefore, the far advanced tumors tended to show membranous and cytoplasmic EGFR overexpression more frequently than the surgically resected tumors, although the difference was not significant.

When these cases were stratified according to histological grade, higher grade (Grades 2 and 3) cancer tissues tended to show membranous EGFR overexpression more frequently (12 of $32,38 \%$ ) than the lower grade (Grade 1) cancer tissues (1 of $12,8 \%$ ) in the surgically resected pancreatic cancers, although the difference was statistically marginal $(\mathrm{P}=0.07)$. The percentage of cytoplasmic EGFR overexpression did not differ statistically between the low grade (Grade 1) tumors (1 of 12, $8 \%$ ) and higher grade (Grades 2 and 3) tumors (9 of 32, 28\%) in the surgically resected cases.

The tissues of the far advanced cancers showed similar rates of membranous and cytoplasmic overexpressions, regardless of histological grade. In the 40 far advanced tumors, membranous EGFR overexpression was detected in $3(43 \%)$ of 7 Grade 1 cases and in $16(48 \%)$ of Grade 2 or 3 cases. In these far advanced tumors, cytoplasmic overexpression of EGFR was detected in 14\% (1 of 7) of Grade 1 tumors and 36\% (12 of 33) of Grade 2 or 3 tumors (Table III).

\section{Discussion}

In the present study, we demonstrated that EGFR overexpression in the cell membrane and cytoplasm was common in both surgically resected and far advanced pancreatic carcinomas. The occurrences of membranous and cytoplasmic EGFR overexpression tended to be higher in the tumors at far advanced stages than in the tumors that were at surgically resectable stages as determined using a global standard kit for EGFR assay.

Cytoplasmic EGFR expression in the far advanced cancers may be explained by the hypothesis of epithelial-tomesenchymal transition which is thought be an important mechanism for promoting cancer invasion and metastasis (23). Persistently activated EGFR can decrease intercellular adhesion between tumor cells and enhance cancer cell migration. Willmarth et al showed that EGF-activated EGFR in MCF10A cells enhanced signal transduction predominantly from the endosomes rather than from the membrane (24). Barr et al (25) suggested that continuously EGF-treated EGFR induced endocytosis of E-cadherin, a cell-to-cell adhesion protein, which enhanced invasiveness in several human cancer cell lines. Ueda et al previously reported that EGFR overexpression in the cytoplasm of pancreatic cancers was associated with poorer clinical outcome of patients $(10,26)$. The present study corroborated that not only membranous overexpression but also cytoplasmic overexpression of EGFR is important for the acquisition of highly aggressive and metastatic properties of pancreatic carcinomas.

In the present study, the rate of EGFR overexpression in surgically resected cancers tended to be higher in higher grade (Grades 2 and 3) tumors than in low grade (Grade 1) tumors. It is not surprising that poorly differentiated pancreatic cancers exhibited a higher incidence of EGFR overexpression as the patients with pancreatic carcinoma with altered EGFR activity tend to show a more aggressive clinical course and a poorer clinical outcome (27). Aggressive tumors appear to require the activation of an EGFR-mediated autocrine signaling in order to maintain proliferation. Therefore, we suppose the possibility that cytoplasmic EGFR protein, which is newly synthesized within the endoplasm reticulum, would be processed at the cellular surface. Some investigators reported that binding of EGF to EGFR activates its receptor tyrosine kinases and accelerates its internalization through clathrin-coated pits followed by the efficient lysosomal targeting of internalized receptors, which results in receptor downregulation and degradation. Thus, the ligand-induced internalization of EGFR, so-called endocytosis trafficking, is characterized as activated EGFR (28-30). If the EGFR ligands dissociated EGFR localized in endosomes, EGFR would be deactivated and recycled to the plasma membrane.

We should consider the possibility that EGFR localization and its activity in advanced or metastatic pancreatic cancers may be modulated by chemotherapy or radiation therapy which those patients had received. It is known that ionizing radiation, hypoxia and oxidative stress can also phosphorylate EGFR with ligand independence, which is sequentially internalized and shuttled into the nucleus (31). Li et al (32) reported that the non-small cell lung cancer $\mathrm{H} 226$ cells which acquire resistance to cetuximab, an anti-EGFR antibody, showed decreased membranous EGFR accompanied by EGFR expressed with nuclear localization. These findings imply that EGFR localization of cancer cells may be an important determinant of responsiveness to specific therapies.

In conclusion, we demonstrated using immunohistochemistry that membranous and cytoplasmic EGFR overexpression was frequently noted in surgically resected and far advanced pancreatic cancers. These findings suggest that membranous and cytoplasmic overexpression of EGFR may be indicative of the potential aggressiveness of pancreatic cancers.

\section{Acknowledgements}

This study was supported in part by a grant-in-aid for defense medicine from the Ministry of Defense and a grant-in-aid from the Foundation for Promotion of Defense Medicine. The authors thank Dr Tatsuro Takahashi and Mr. Ryuji Saito, Department of Central Laboratory, Japan Labor Health and Welfare Organization Kushiro Rosai Hospital, Kushiro, Hokkaido, Japan, for technical assistance with the immunohistochemistry.

\section{References}

1. Matsuno S, Egawa S, Fukuyama S, et al: Pancreatic Cancer Registry in Japan: 20 years of experience. Pancreas 28: 219-230, 2004.

2. Bramhall SR, Allum WH, Jones AG, Allwood A, Cummins C and Neoptolemos JP: Treatment and survival in 13,560 patients with pancreatic cancer, and incidence of the disease, in the West Midlands: an epidemiological study. Br J Surg 82: 111-115, 1995.

3. Parkin DM, Bray FI and Devesa SS: Cancer burden in the year 2000. The global picture. Eur J Cancer 37 (Suppl 8): S4-S66, 2001. 
4. Burris HA III, Moore MJ, Andersen J, et al: Improvements in survival and clinical benefit with gemcitabine as first-line therapy for patients with advanced pancreas cancer: a randomized trial. J Clin Oncol 15: 2403-2413, 1997.

5. Oettle H, Post S, Neuhaus P, et al: Adjuvant chemotherapy with gemcitabine vs observation in patients undergoing curativeintent resection of pancreatic cancer: a randomized controlled trial. JAMA 297: 267-277, 2007.

6. Ueno H, Kosuge T, Matsuyama Y, et al: A randomised phase III trial comparing gemcitabine with surgery-only in patients with resected pancreatic cancer: Japanese Study Group of Adjuvant Therapy for Pancreatic Cancer. Br J Cancer 101: 908-915, 2009.

7. Kindler HL, Friberg G, Singh DA, et al: Phase II trial of bevacizumab plus gemcitabine in patients with advanced pancreatic cancer. J Clin Oncol 23: 8033-8040, 2005.

8. Moore MJ, Goldstein D, Hamm J, et al: Erlotinib plus gemcitabine compared with gemcitabine alone in patients with advanced pancreatic cancer: a phase III trial of the National Cancer Institute of Canada Clinical Trials Group. J Clin Oncol 25: 1960-1966, 2007.

9. Salomon DS, Brandt R, Ciardiello F and Normanno N: Epidermal growth factor-related peptides and their receptors in human malignancies. Crit Rev Oncol Hematol 19: 183-232, 1995.

10. Ueda S, Ogata S, Tsuda H, et al: The correlation between cytoplasmic overexpression of epidermal growth factor receptor and tumor aggressiveness: poor prognosis in patients with pancreatic ductal adenocarcinoma. Pancreas 29: e1-e8, 2004.

11. Mendelsohn J and Baselga J: Status of epidermal growth factor receptor antagonists in the biology and treatment of cancer. J Clin Oncol 21: 2787-2799, 2003.

12. Cappuzzo F, Hirsch FR, Rossi E, et al: Epidermal growth factor receptor gene and protein and gefitinib sensitivity in non-small cell lung cancer. J Natl Cancer Inst 97: 643-655, 2005.

13. Hirsch FR, Varella-Garcia M, McCoy J, et al: Increased epidermal growth factor receptor gene copy number detected by fluorescence in situ hybridization associates with increased sensitivity to gefitinib in patients with bronchioloalveolar carcinoma subtypes: a Southwest Oncology Group Study. J Clin Oncol 23: 6838-6845, 2005.

14. Tsao MS, Sakurada A, Cutz JC, et al: Erlotinib in lung cancer molecular and clinical predictors of outcome. N Engl J Med 353 133-144, 2005

15. Lynch TJ, Bell DW, Sordella R, et al: Activating mutations in the epidermal growth factor receptor underlying responsiveness of non-small cell lung cancer to gefitinib. N Engl J Med 350: 2129-2139, 2004

16. Han SW, Kim TY, Hwang PG, et al: Predictive and prognostic impact of epidermal growth factor receptor mutation in non-small cell lung cancer patients treated with gefitinib. J Clin Oncol 23 : 2493-2501, 2005.

17. Mitsudomi T, Kosaka T, Endoh H, et al: Mutations of the epidermal growth factor receptor gene predict prolonged survival after gefitinib treatment in patients with non-small cell lung cancer with postoperative recurrence. J Clin Oncol 23 2513-2520, 2005.
18. Paez JG, Janne PA, Lee JC, et al: EGFR mutations in lung cancer: correlation with clinical response to gefitinib therapy. Science 304: 1497-1500, 2004.

19. Takano T, Ohe Y, Sakamoto H, et al: Epidermal growth factor receptor gene mutations and increased copy numbers predict gefitinib sensitivity in patients with recurrent non-small cell lung cancer. J Clin Oncol 23: 6829-6837, 2005.

20. Takano T, Ohe Y, Tsuta K, et al: Epidermal growth factor receptor mutation detection using high-resolution melting analysis predicts outcomes in patients with advanced non-small cell lung cancer treated with gefitinib. Clin Cancer Res 13: 5385-5390, 2007.

21. Sobin LH and Wittekind Ch (eds): International Union Against Cancer (UICC). TNM Classification of Malignant Tumors. 6th edition. Wiley-Liss, New York, 2002.

22. Tsuda H, Akiyama F, Terasaki H, et al: Detection of HER-2/neu (c-erb B-2) DNA amplification in primary breast carcinoma. Interobserver reproducibility and correlation with immunohistochemical HER-2 overexpression. Cancer 92: 2965-2974, 2001.

23. Dembinski JL and Krauss S: Characterization and functional analysis of a slow cycling stem cell-like subpopulation in pancreas adenocarcinoma. Clin Exp Metastasis 26: 611-623, 2009.

24. Willmarth NE, Baillo A, Dziubinski ML, Wilson K, Riese DJ II and Ethier SP: Altered EGFR localization and degradation in human breast cancer cells with an amphiregulin/EGFR autocrine loop. Cell Signal 21: 212-219, 2009.

25. Barr S, Thomson S, Buck E, et al: Bypassing cellular EGF receptor dependence through epithelial-to-mesenchymal-like transitions. Clin Exp Metastasis 25: 685-693, 2008.

26. Ueda S, Hatsuse K, Tsuda H, et al: Potential crosstalk between insulin-like growth factor receptor type 1 and epidermal growth factor receptor in progression and metastasis of pancreatic cancer. Mod Pathol 19: 788-796, 2006.

27. Holbro T, Civenni G and Hynes NE: The ErbB receptors and their role in cancer progression. Exp Cell Res 284: 99-110, 2003.

28. Madshus IH and Stang E: Internalization and intracellular sorting of the EGF receptor: a model for understanding the mechanisms of receptor trafficking. J Cell Sci 122: 3433-3439, 2009.

29. Sorkin A and Goh LK: Endocytosis and intracellular trafficking of ErbBs. Exp Cell Res 315: 683-696, 2009.

30. Sorkin A and von Zastrow M: Endocytosis and signalling: intertwining molecular networks. Nat Rev Mol Cell Biol 10: 609-622, 2009.

31. Dittmann K, Mayer C, Kehlbach R and Rodemann HP: Radiation-induced caveolin-1 associated EGFR internalization is linked with nuclear EGFR transport and activation of DNA-PK. Mol Cancer 7: 69, 2008.

32. Li C, Iida M, Dunn EF, et al: Nuclear EGFR contributes to acquired resistance to cetuximab. Oncogene 28: 3801-3813, 2009. 